\title{
Performance of the MCP-PMTs for the TOP counter in the Belle II experiment
}

\author{
T. Yonekura ${ }^{* a}$, Y. Ito, ${ }^{a}$ K. Inami, ${ }^{a}$ K. Suzuki, ${ }^{a}$ S. Hirose,${ }^{a}$ D. Furumura,${ }^{a}$ T. lijima, ${ }^{b}$ Y. \\ Kato, ${ }^{b}$ Y. Sato, ${ }^{b}$ T. Hayakawa, ${ }^{b}$ K. Matsuoka, ${ }^{b}$ \\ ${ }^{a}$ Department of Physics, Nagoya University \\ Furo-cho, Chikusa-ku, Nagoya, Japan \\ ${ }^{b}$ KMI, Nagoya University \\ Furo-cho, Chikusa-ku, Nagoya, Japan \\ E-mail: yonekura@hepl.phys . nagoya-u.ac.jp
}

\begin{abstract}
We developed the micro-channel-plate (MCP) PMT for the Time-of-Propagation (TOP) counter, which is a novel Cherenkov counter to be used for particle identification in the Belle II experiment. The MCP-PMT has excellent performance for single photon detection; a transit time spread (TTS) better than about $40 \mathrm{ps}$ and a nominal gain as high as $2 \times 10^{6}$. For the Belle II TOP counter, 512 MCP-PMTs are used. We started mass production of the MCP-PMT and measured the TTS, gain and quantum efficiency of every MCP-PMT to control the quality. Since the MCP-PMT is used in $1.5 \mathrm{~T}$, the TTS and gain were measured with and without the magnetic field. The gain on each anode was found to be different from each other by up to a factor of two in some of the MCP-PMTs. We found that the gain drops by $20-80 \%$ in a $1.5 \mathrm{~T}$. Regarding the lifetime of the MCP-PMT, we succeeded in extending the lifetime significantly by introducing an atomic layer deposition technique on the MCP coating. The lifetime of the conventional MCP-PMT is about $1 \mathrm{C} / \mathrm{cm}^{2}$ and the one of ALD-MCP-PMT is $3 \mathrm{C} / \mathrm{cm}^{2}$ or longer.
\end{abstract}

Technology and Instrumentation in Particle Physics 2014,

2-6 June, 2014

Amsterdam, the Netherlands

\footnotetext{
* Speaker.
} 


\section{Introduction}

The TOP (Time Of Propagation) counter [1-4] is a novel ring imaging Cherenkov detector which mainly consists of a quartz radiator bar and photon detectors as shown in Fig 1. It is installed in the barrel region of the Belle II detector[5] for particle identification. Cherenkov photons generated by a pion or kaon propagate in the quartz and are measured by photon detectors at the end of the quartz. The number of detected photons is about 20. The difference of the time of propagation between a $3 \mathrm{GeV} / \mathrm{c}$ pion and kaon is about $100 \mathrm{ps}$. The TOP counter identifies a pion and kaon by measuring the difference. Therefore it is necessary for the photon detector to have a transit time spread (TTS) less than 50 ps and a high detection efficiency. In addition, because the background rate is estimated to be as high as several MHz per photon detecter in the Belle II luminosity frontier experiment and the TOP counter is used in $1.5 \mathrm{~T}$, the photon detector has to have a long lifetime and work in $1.5 \mathrm{~T}$. Therefore we use the MCP (Micro-Channel-Plate)-PMT [6-9] for the TOP counter. The requirements for the MCP-PMT are the followings: the TTS less than $50 \mathrm{ps}$, enough gain to detect single photon and the quantum efficiency (QE) greater than $24 \%$ and $28 \%$ on average at the peak wavelength.

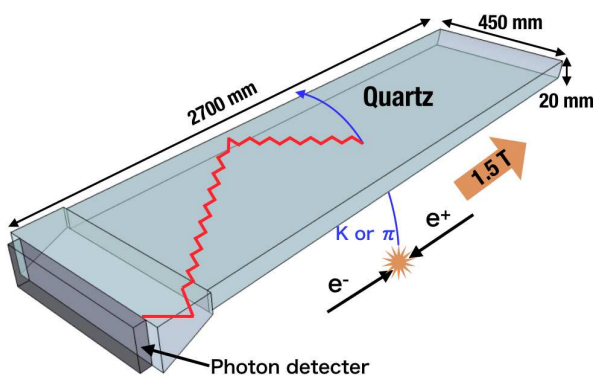

Figure 1: Schematic view of the TOP counter. An example of a charged particle from the interaction point and a Cherenkov photon propagating in the quartz bar is drawn. The magnetic field direction is indicated by the arrow.

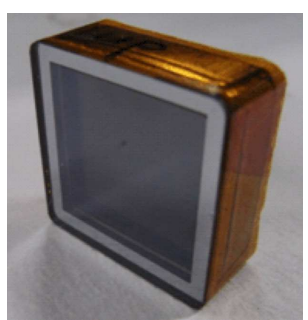

Figure 2: Photograph of the MCP-PMT.

The MCP-PMT was developed at Nagoya University and Hamamatsu photonics K.K. Fig 2 is a photocathode of the MCP-PMT. The MCP-PMT is square in shape to minimize the dead region down to about $30 \%$. The height and width is $27.6 \mathrm{~mm}$ and the thickness is $13.1 \mathrm{~mm}$. The number of channels are 16. Figure 3 is a cross section view of the MCP-PMT. The photoelectron is multiplied in the micro channels of the two MCPs. The MCP-PMT has two $400 \mu \mathrm{m}$ thick MCPs made of lead glass. The diameter of the micro channel is $10 \mu \mathrm{m}$ and the aperture ratio is about $60 \%$. The bias angle of the micro channel is 13 degrees. We use two types of MCP: a conventional MCP and an MCP with atomic layer deposition (ALD) coating. The ALD-MCP was recently adapted to extend the lifetime of the photocathode. We succeeded in developing the MCP-PMT which satisfied the requirements and started mass production of 512 MCP-PMTs for the TOP counter. In order to produce 512 MCP-PMTs which satisfy the requirements, we check the performance of every MCP-PMT and feed back to the mass production. Three measurements are performed to 


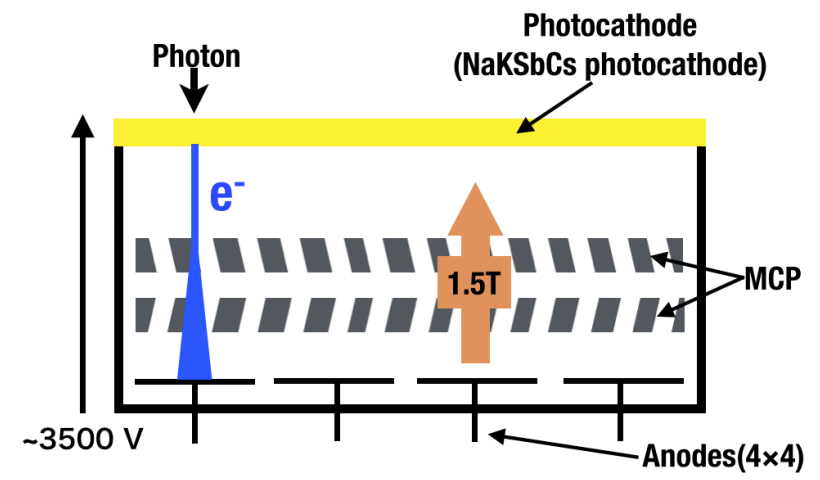

Figure 3: Schematic cross section view of the MCP-PMT.

check the performance: laser test both in 0 and $1.5 \mathrm{~T}$ to check the gain and TTS, QE measurement and lifetime sampling test.

\section{Measurement of the gain and TTS}

The gain and TTS are measured in $0 \mathrm{~T}$ and $1.5 \mathrm{~T}$. The gain of the MCP-PMT drops in $1.5 \mathrm{~T}$ due to the following two reasons. In $1.5 \mathrm{~T}$ the electrons hit the MCP in a shorter distance or lower energy than in $0 \mathrm{~T}$ because the trajectory is changed by Lorentz force. Therefore the number of secondary electrons is reduced. Another reason is that the electrons are converged in several micro channels of the second MCP in 1.5 T and the amplification in the second MCP saturates. The gain drop in $1.5 \mathrm{~T}$ makes the signal-to-noise ratio lower and TTS worse. Therefore it is also necessary to measure the gain and TTS in $1.5 \mathrm{~T}$. The gain and TTS in $0 \mathrm{~T}$ are measured at several high voltages (HVs). The measurement in $1.5 \mathrm{~T}$ is performed at one $\mathrm{HV}$ to check the performance change in 1.5 T. The HV dependence of the gain and TTS is the same in $0 \mathrm{~T}$ and $1.5 \mathrm{~T}$.

\subsection{Setup}

The laser test setup in $1.5 \mathrm{~T}$ is shown in Fig 4. The laser head, filter, spot and MPPC are moved by the stage, and we scan the all channel of the MCP-PMT in $1.5 \mathrm{~T}$. The gain and TTS for single photon are measured with a pico-second pulse laser. The laser intensity is reduced by an ND filter followed by two slits. The slits make a laser spot of about $1 \mathrm{~mm}$ diameter on the MCP-PMT. The laser is divided by a beam splitter to monitor the intensity with an MPPC.

The magnetic field is applied with a dipole magnet shown in Fig 5. The laser part is connected by a G10 arm to a moving stage put away from the magnet. So that the laser position on the MCP-PMT can be changed automatically. The hit timing and the output charge are measured by a CAMAC TDC and ADC, respectively.

\subsection{Result of the gain in $1.5 \mathrm{~T}$}

Figure 6 is an example of the ADC distribution for single photon. The gain is defined as the mean of the ADC distribution. The ADC distribution becomes sharp in $1.5 \mathrm{~T}$. Because the low and high number of signal decrease in $1.5 \mathrm{~T}$. Figure 7 shows the relative gain in $1.5 \mathrm{~T}$ to $0 \mathrm{~T}$ for each 


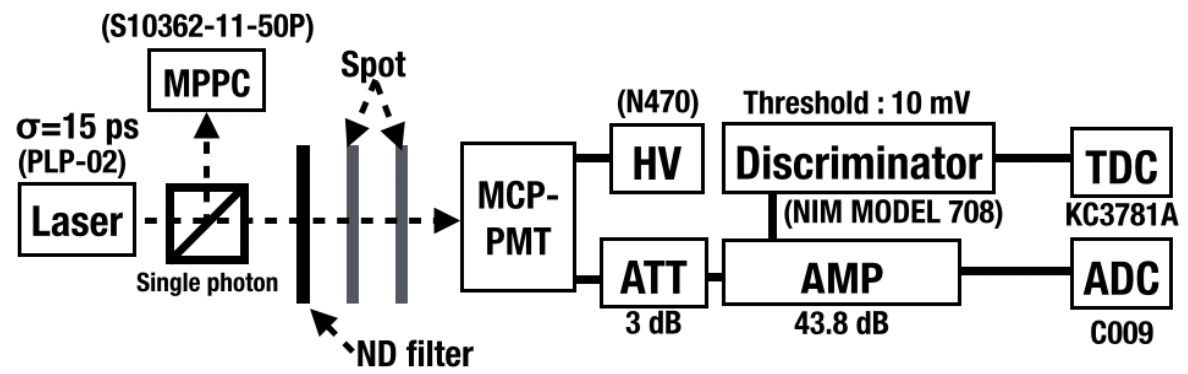

Figure 4: Schematic of the setup for the laser test in $1.5 \mathrm{~T}$.

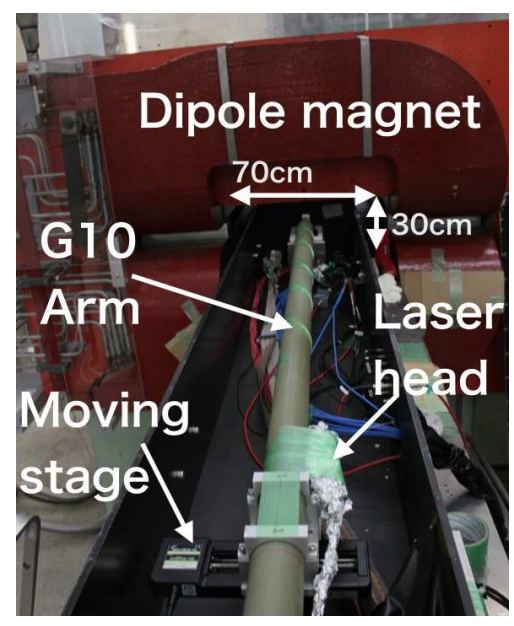

Figure 5: Photograph of the setup for the laser test in 1.5 T. The moving stage is put in the dark box outside of the magnet. The MCP-PMT is set in the slit of the dipole magnet.

channel. The relative gain of the ALD type is smaller than the conventional one. However the gain of ALD in $1.5 \mathrm{~T}$ is greater than $5 \times 10^{5}$, which is enough to detect single photon.

\subsection{Result of the TTS in $1.5 \mathrm{~T}$}

Figure 8 is an example of the TDC distribution corrected for the time walk effect using the correlation between the TDC and ADC. We fit the distribution with a double Gaussian, and the TTS is defined as the standard deviation of the primary Gaussian. The tail is due to the recoil of the photoelectron on the first MCP surface. Figure 9 shows the TTS in $1.5 \mathrm{~T}$ for each channel. Every channel has a better TTS than the requirement of $50 \mathrm{ps.}$

\section{QE measurement}

\subsection{Setup for the QE measurement}

We measure the current on the photocathode $\left(I_{M C P-P M T}\right)$ and the silicon photodiode $\left(I_{P D}\right)$ with a picoammeter, and the $\mathrm{QE}$ of the MCP-PMT $\left(Q E_{M C P-P M T}\right)$ is calculated as 


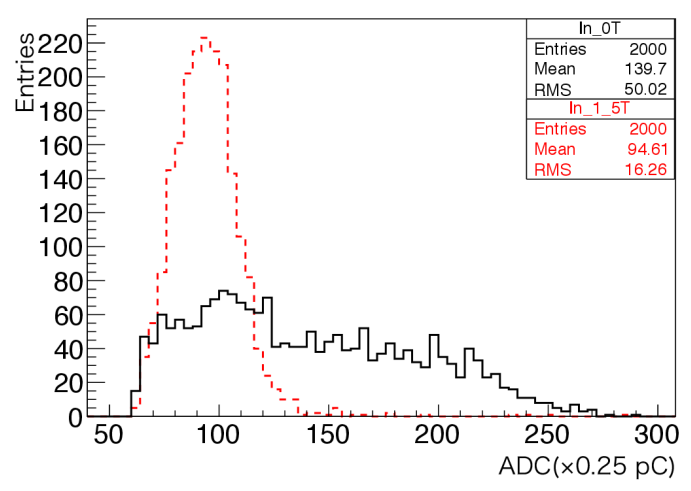

Figure 6: ADC distribution of the MCP-PMT. The solid line is the ADC distribution in $0 \mathrm{~T}$. The dotted line is the one in $1.5 \mathrm{~T}$.

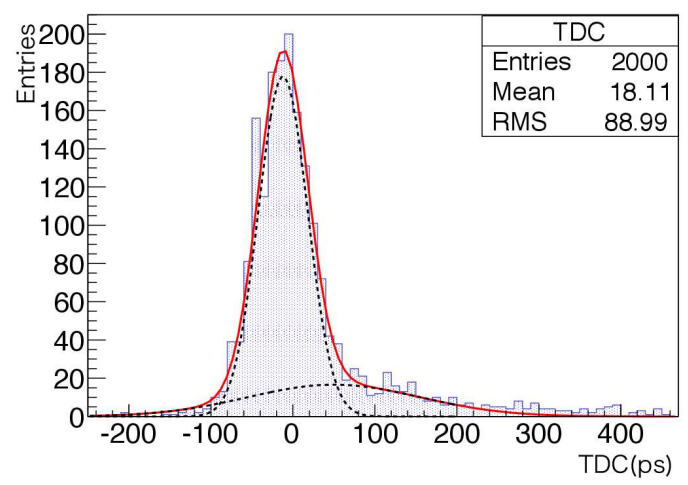

Figure 8: TDC distribution of the MCP-PMT in $1.5 \mathrm{~T}$. The filled histogram is the TDC distribution. The solid line is a double Gaussian fitted to the distribution. The dashed line shows each Gaussian component.

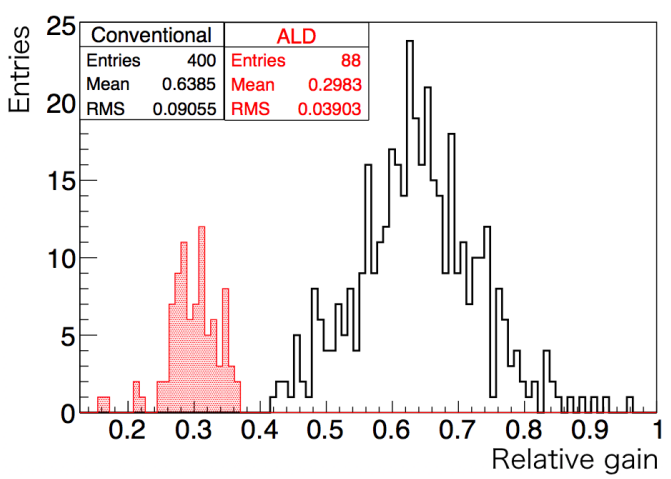

Figure 7: The relative gain $(1.5 \mathrm{~T} / 0 \mathrm{~T})$. The black histogram is the conventional MCP-PMT data. The filled histogram is the ALD-MCP-PMT data.

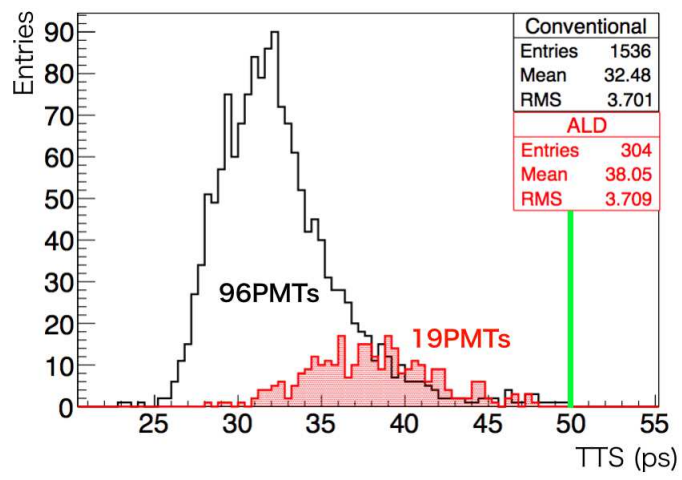

Figure 9: TTS in $1.5 \mathrm{~T}$ for 96 conventional MCPPMTs (black histogram) and 19 ALD-MCP-PMTs (filled histogram). The line is the requirement of the TTS.

$$
Q E_{M C P-P M T}=\frac{I_{M C P-P M T}}{I_{P D}} \times Q E_{P D},
$$

where $Q E_{P D}$ is the $\mathrm{QE}$ of the photodiode. Figure 10 shows the setup for the $\mathrm{QE}$ measurement. We use a Xe lamp and a spectroscope to select the wavelength. We replace a sharp cut filter depending on the wavelength. The spot light made by a slit is irradiated to the MCP-PMT and the photodiode on a moving stage.

\subsection{Result of the QE measurement}

Figure 11 shows the QE scanned over the photocathode to check the uniformity. The QE is uniformly in the photocathode surface, and is lower in a place that is not a photocathode surface. 


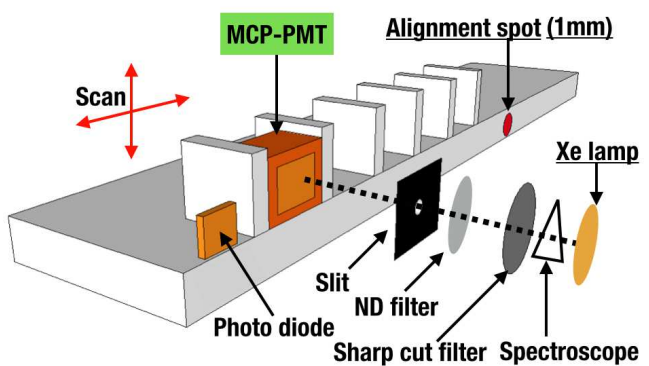

Figure 10: The setup for the $\mathrm{QE}$ measurement.

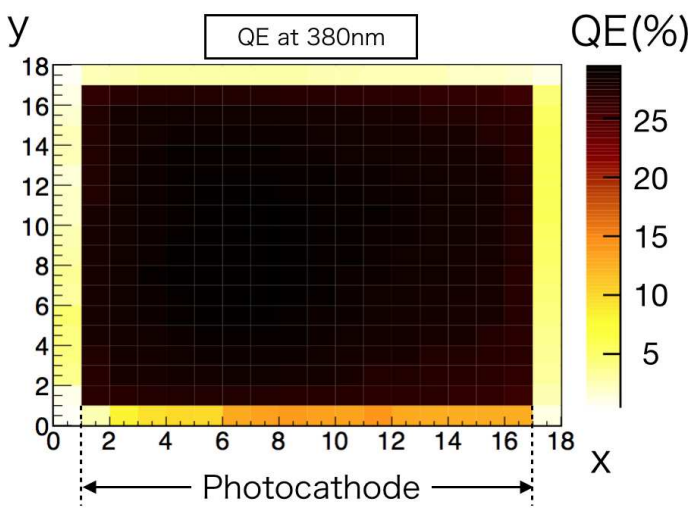

Figure 11: QE of the MCP-PMT scanned over the photocathode. The lower QE points are not over the photocathode.

Figure 12 shows the result of QE measurement as a function of the wavelength. The peak wavelength is about $360 \mathrm{~nm}$. The measured QE is consistent with the one by HAMAMATSU except for the wavelength below $400 \mathrm{~nm}$ because the setup of Hamamatsu and Nagoya is different. Therefore, the result is different systematically. Figure 13 is the QE at the wavelength of $360 \mathrm{~nm}$ for 474 MCP-PMTs. The average is $28.7 \%$. It meets our requirement. For the two MCP-PMTs whose $\mathrm{QE}$ at $360 \mathrm{~nm}$ is less than $24 \%$, the QE peaks at $420 \mathrm{~nm}$ and it is greater than $24 \%$. The peak wavelength is different less than $24 \%$. It meet our requirement in the peak wavelength.

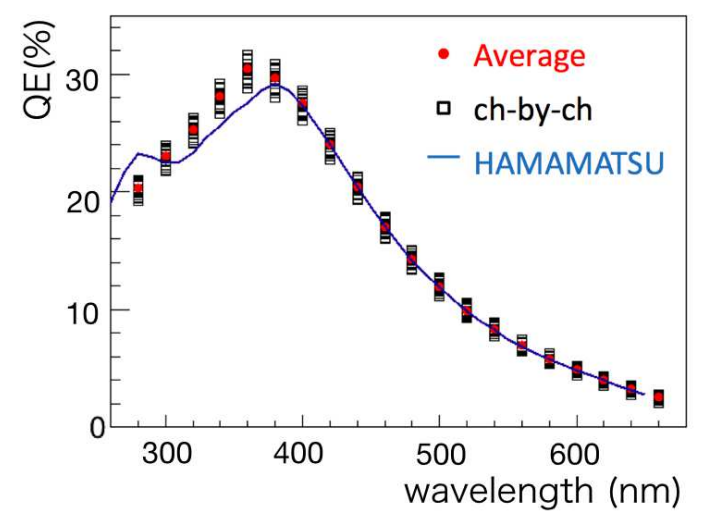

Figure 12: QE of the MCP-PMT as a function of the wavelength. The solid line is the HAMAMATSU measurement data. The circle plot is the average of the measurement in Nagoya. The square plot is the measurement data of ch by ch in Nagoya.

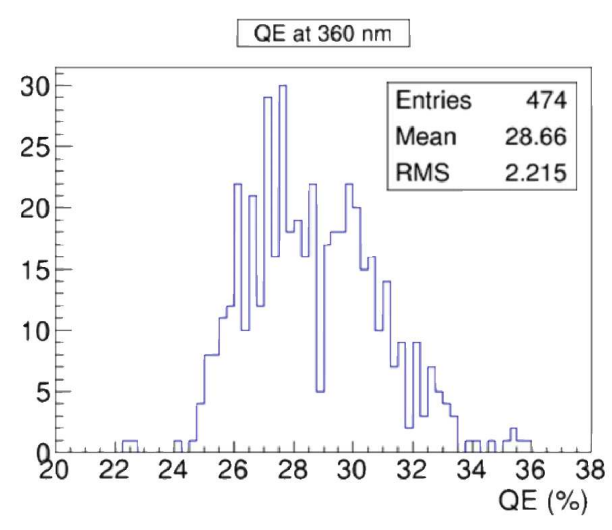

Figure 13: $Q E$ at the wavelength of $360 \mathrm{~nm}$ for 474 MCP-PMTs. 


\section{Lifetime test}

\subsection{Lifetime of the photocathode}

The photocathode is degraded by neutral gas and feed back ions desorbed from the MCPs by the multiplied electrons. We put ceramic blocks and an aluminum film on the second MCP surface to prevent the gas and ions from reaching the photocathode as shown in Fig 14. Because the QE drops as a function of the integrated output charge, we define the lifetime of the photocathode as the output charge where the $\mathrm{QE}$ decreases to $80 \%$ of the beginning. The integrated output charge in Belle II is expected to be 1.5 to $3 \mathrm{C} / \mathrm{cm}^{2}$ at the $5 \times 10^{5}$ gain. We checked the lifetime of the conventional and ALD MCP-PMT. We expect a longer lifetime for the ALD type because of less out gas and ions.

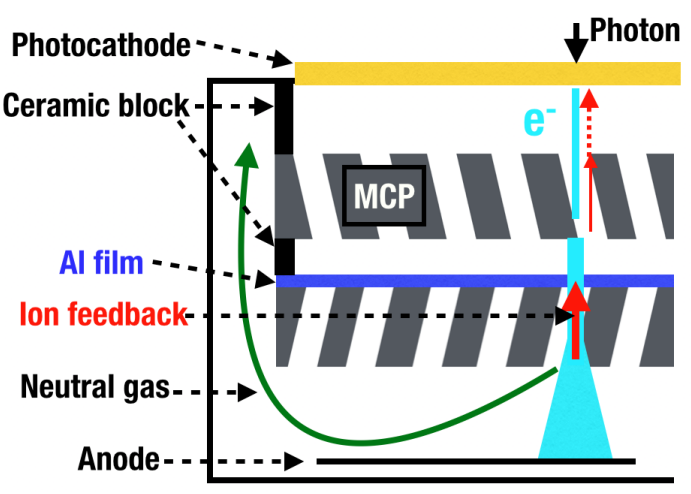

Figure 14: Schematic cross section view of the MCPPMT showing the ceramic block and the Al film for the lifetime improvement.

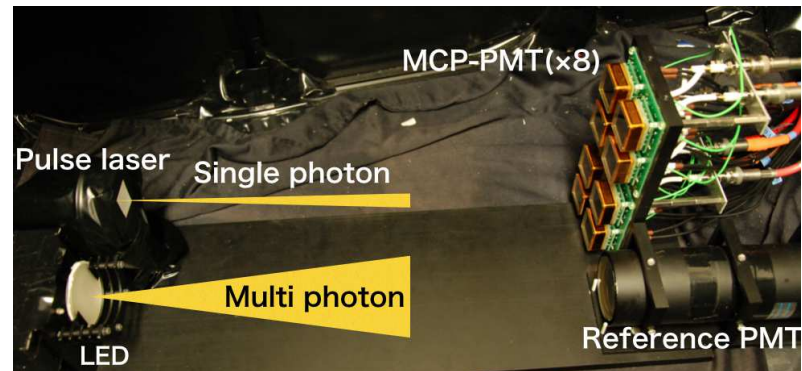

Figure 15: Setup of the lifetime test

\subsection{Setup of the lifetime test}

Figure 15 shows the setup for the lifetime test. We degrade the photocathode of the MCP-PMT with LED light. The integrated output charge is about $1 \mathrm{C} / \mathrm{cm}^{2}$ per month. The output charge is measured by a CAMAC ADC. We monitor the $\mathrm{QE}$ by measuring the relative hit rate to a reference PMT.

\subsection{Result of the lifetime test}

Figure 16 is the result of the lifetime test. The lifetime of the conventional MCP is about 0.5 to $2 \mathrm{C} / \mathrm{cm}^{2}$. Since it is less than the expected output charge in Belle II, the conventional MCP-PMTs need to be replaced with new ones during the operation. The ALD-MCP-PMT has a longer lifetime than the conventional one. However one of the ALD has a lifetime of $3 \mathrm{C} / \mathrm{cm}^{2}$, which is the same as the integrated output charge in Belle II. Therefore we are still trying to improve the lifetime further. 


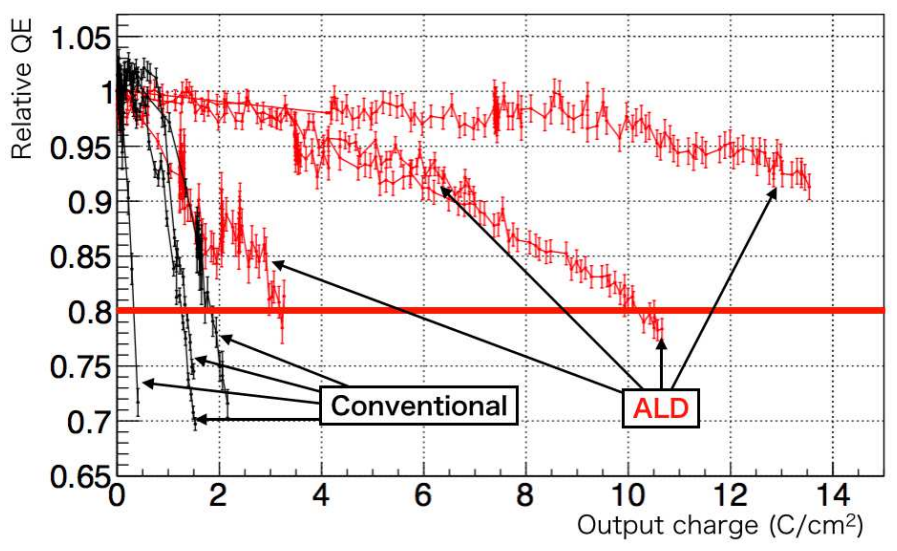

Figure 16: Result of the lifetime test. The horizontal axis is the integrated output charge and the vertical axis is the relative $\mathrm{QE}$. The line is the requirement of the lifetime.

\section{5. summary}

The MCP-PMT mass production is successfully on going. The QE is $28.7 \%$ on average at $360 \mathrm{~nm}$. The gain in $1.5 \mathrm{~T}$ is high enough to detect single photon and the TTS in $1.5 \mathrm{~T}$ is about 40 ps. These performance satisfy the requirements for TOP counter. The MCP-PMT is required to have a longer lifetime than $1.5-3 \mathrm{C} / \mathrm{cm}^{2}$. The lifetime of the conventional MCP-PMT is about 0.5 to $2 \mathrm{C} / \mathrm{cm}^{2}$. By introducing the ALD-MCP, the lifetime is improved to $3 \mathrm{C} / \mathrm{cm}^{2}$ or longer.

\section{Acknowledgments}

This work was supported by MEXT Grant-in-Aid for Scientic Research on Innovative Areas "Elucidation of NewHadrons with a Variety of Flavors". We also thank Electron Tube Devision, Hamamatsu Photonics K.K. for discussion and providing excellent products for us.

\section{References}

[1] M. Akatsu, et al., Nuclear Instruments and Methods in Physics Research Section A 440 (2000) 124.

[2] T. Oshima, ICFA Instrumentation Bulletin 20 (2000) 2.

[3] T. Oshima, Nuclear Instruments and Methods in Physics Research Section A 453 (2000) 331.

[4] Y. Enari, et al., Nuclear Instruments and Methods in Physics Research Section A 494 (2002) 430.

[5] T. Abe, et al., KEK-REPORT-2010-1, 2010.

[6] M. Akatsu, et al., Nuclear Instruments and Methods in Physics Research Section A 528 (2004) 763.

[7] N. Kishimoto, et al., Nuclear Instruments and Methods in Physics Research Section A 564 (2006) 204.

[8] K. Inami, et al., Nuclear Instruments and Methods in Physics Research Section A 592 (2008) 247.

[9] T. Jinno, et al., Nuclear Instruments and Methods in Physics Research Section A 629 (2011) 111. 